\title{
Estrategias metodológicas para el desarrollo lógico-matemático Nivel I ciclo de Educación Inicial
}

Elba María Urbina Espinoza, Daysi Marlene Rodríguez Canales.

Tutora: Dra. Valinda Sequeira Calero.

Facultad De Educación e Idiomas

\section{RESUMEN}

La investigación "Estrategias metodológicas para el desarrollo lógico-matemático del niño Carlos José del III nivel del I ciclo de Educación Inicial en el Centro Escolar Santa Rosa, Barrio Santa Rosa ubicado en el distrito VI del municipio de Managua en el I semestre, del año 2014" se realizó bajo el enfoque investigación-acción el cual se constituye de diferentes etapas. Esto nos permitió familiarizarnos, observar, caracterizar, explorar, indagar, diagnosticar, reflexionar y proponer diferentes estrategias que pueden ser incluidos en el sistema educativo para poder dar respuestas a las necesidades del niño. A través del diagnóstico se identificó lo siguiente: el niño presentaba dificultades en el desarrollo lógico- matemático; la docente no muestra interés en las necesidades del niño quien es ignorado y no apoyado en su proceso de enseñanza aprendizaje. Moomaw, (2011), comparte que los niños que aprenden los fundamentos de matemáticas en la etapa preescolar tienen las mejores posibilidades de logros escolares. Esto nos facilitó el planteó de objetivos y estrategias que se aplicaron con el propósito de superar dificultades observadas, desarrollando actividades dirigidas a la seriación, agrupación y clasificación, que ayudaran al niño en el desarrollo de su proceso lógico- matemático. Los resultados fueron notorios y nos sensibilizó más con nuestro trabajo hacia los niños, obtuvimos sentido de comprensión y compromiso al desempeñar un trabajo de calidad con estos individuos en proceso de desarrollo, dándoles un seguimiento adecuado con el estímulo, la comprensión, el sentido de pertenencia, dedicación, preparación y la autoevaluación.

Palabras claves: estrategias, desarrollo, matemática

\section{SUMMARY}

The research "Methodological strategies for logical-mathematical development of the child José Carlos I of III level early childhood education cycle in Santa Rosa School, in Santa Rosa neighborhood located in the sixth district of the municipality of Managua in the first half of the year 2014 " it was held under the action research approach which is made up of different stages. This allowed us to familiarize ourselves to observe, characterize, explore, investigate, diagnose, reflect and propose different strategies that can be included in the educational system to provide answers to the needs of the child. Through diagnosis identified the following: the child had difficulties in logical-mathematical development; the teacher is not interested in the needs of the child who is ignored and not supported in his process of learning. Moomaw, (2011), shares that children who learn the basics of math in preschool have the best chances of school achievement. This provided us with the proposed objectives and strategies to apply them in order to overcome difficulties found by developing activities for serialization, grouping and sorting, to assist the child in developing his mathematical logical process. At the end, the results were noticeable and this situation sensitized us with our work towards children, we had a better comprehension and commitment to perform a quality work with these kind of children in process of development, giving them 
appropriate monitoring encouragement, understanding, sense of belonging, dedication, preparation and selfevaluation.

Keywords: strategies, development, mathematic

\section{INTRODUCCIÓN}

En la segunda semana de visita al centro, pudimos identificar al niño Carlos José luego de haber aplicado una hoja de trabajo para todos los niños, en la cual se les orientaba realizar actividades para evaluar el conocimiento que ellos tenían en los conceptos básicos tales como: izquierda, derecha, arriba, abajo y la identificación de colores. Además de eso, nos llamó la atención la necesidad que el niño nos transmitía de falta de apoyo por parte de la docente, dado que en la mayoría de las observaciones pudimos constatar que el niño era un alumno fantasma dentro del aula.

Carlos José es un niño de 5 años que estudia en el Centro Escolar Santa Rosa, ubicado en el barrio del mismo nombre en la ciudad de Managua. Vive con sus padres y sus hermanos. De escasos recursos económicos. Es amoroso y dinámico. Por primera vez asiste al colegio. Su madre logró terminar el sexto grado de educación primaria ahora solamente es ama de casa y su padre es analfabeta y trabaja de vigilante. En su hogar viven 15 personas en total. Sus hermanos mayores son drogadictos y alcohólicos, por tanto el niño no tiene ningún ejemplo de superación debido a las condiciones económicas y familiares en las que vive.

Para comprender el caso se realizó un diagnóstico, el cual arrojo lo siguiente:

El niño mostraba dificultades en reconocer colores; identificar conceptos básicos tales como: izquierda-derecha, arriba-abajo, adelante-atrás- en medio, grande- pequeño- mediano, encima de- debajo de; tomaba incorrectamente la crayola; no realizaba ejercicios de seriación; coloreaba saliéndose del espacio dado; no seguía orientaciones de secuencias lógicas; su coordinación ojo-mano no era la adecuada, dejaba sus asignaciones incompletas y se distraía con facilidad.

La importancia del proceso lógico- matemático radica en que la formación temprana de este componente, entre los 0 y 6 años. Es decir, en la Educación Inicial es tan importante en una sociedad que exige un alto desempeño en los procesos de razonamiento superior. Puesto que el éxito en los estudios subsiguientes y el desempeño en muchas carreras y profesiones depende del desarrollo adecuado de las estructuras cognitivas del individuo. 
Ramírez (2008) expresa que las estrategias metodológicas son aquellas que permiten identificar principios, criterios y procedimientos que configuran la forma de actuar del docente en relación con la programación, implementación y evaluación del proceso de enseñanza-aprendizaje.
a.-Estrategias de Motivación o de iniciación
b.- Estrategias de desarrollo
c.- Estrategias de culminación

\section{d.- La Evaluación}

Brissiaud (1993) plantea que el punto de partida para iniciar el pensamiento lógico es la abstracción de características o propiedades físicas de los objetos para luego comparar unos con otros. La comparación de objetos en función de sus características físicas permite establecer relaciones de semejanza y diferencia, que a su vez son la base para:

a). Ordenarlos según la variación de una de sus magnitudes físicas, a través del proceso de seriación.

b). Ordenarlos en grupos por sus semejanzas, a través del proceso de clasificación.

Cada objeto puede ser explorado para determinar en él características o propiedades.

\section{MATERIAL Y MÉTODO}

El estudio corresponde a un enfoque de investigación-acción, el cual ocurre y se desarrolla cada cierto tiempo y de la misma forma. Además, tiene sus fases: problema o foco de investigación; diagnóstico del problema o estado de la situación e hipótesis de acción o acción estratégica. Estas nos permiten analizar la realidad del problema para saber cómo ocurre; elaborar un plan para crear condiciones que se puedan realizarlas; hacer revisiones para identificar aspectos que debemos mejorar y modificar según los resultados obtenidos, siempre y cuando se ajusten a lo que se investiga. Esto es debido a que esto va más allá de investigar. Conlleva a ser partícipes de las necesidades y soluciones, a establecer nuevas relaciones con otras personas ya que es hecha con y para otra gente, su meta es la mejora personal para la transformación social, esencialmente colaborativa a las cuales también le podemos dar continuidad.

Se aplicaron diversas técnicas tales como: entrevistas a la docente y padres de familia y guías de observación al proceso de enseñanza- aprendizaje y al entorno familiar del niño. Así como también 
planes de acción los cuales tenían el objetivo de que el niño reforzara su proceso en el pensamiento lógico-matemático.

Toda la información que necesitábamos claramente se obtuvo con cada día y semana que pasaba así se recopilaban todas las evidencias de cada actividad que realizábamos las cuales eran a través de:

- Observaciones que nos permitían evaluar el desarrollo del niño y cada una de las dificultades que presentaba dentro del aula de clases, en trabajos individuales y grupales.

- Análisis de hojas de aplicación.

- Fotografías de las distintas actividades y comportamientos del niño en el aula de clases y en su hogar.

- Videos de los trabajos desarrollados con el niño en el colegio y en su casa.

- Diario de campo en el cual se recogía las evidencias escritas por nosotras de lo que se observaba en las diferentes situaciones que se dieron. Esta información se recogía por día y horario de clases, todo esto nos ayudó a estar más claras de la situación educativa en la que el niño estaba y a identificar sus dificultades.

\section{RESULTADOS}

Este tema de investigación plenamente nos hizo posible reflexionar de manera personal la magnitud de la repercusión que tiene para cada individuo en desarrollo y dejó en nosotras una serie de experiencias con mucha riqueza que pudimos hacer propias durante el camino que por ende proporcionó mucha ayuda a nosotras, a la docente, al colegio y la madre del niño. Nuestra finalidad al emplear la teoría era primordialmente obtener un muy buen avance en las habilidades matemáticas del niño de acuerdo a las dificultades encontradas en este proceso. Definitivamente en cada sesión, actividad y semana que pasaba en donde realizábamos los planes de acción propuestos el niño mostró mucho interés y entusiasmo, el niño daba respuesta positiva a cada una de las actividades que le orientábamos, participaba de manera activa, pero lo más importante era que no se rendía ante el hecho de no poder lograr lo que se le proponía, el niño intentaba una y más veces hasta que lo lograba a chorros de sudor pero lo hacía sin presionarlo ni decirle que debía de intentarlo, aceptaba los retos, aunque hubo dos momentos en los cuales la maestra se mostró con actitud negativa y poco colaboradora predisponiendo al niño. Éste no quería realizar las 
actividades solo, mostraba desánimo y negación pero aun así se le orientó que podía trabajar con uno o dos compañeritos y de esta manera sin problema alguno concluimos las actividades.

En otro momento no se pudo realizar el plan de acción en el colegio por motivos ajenos a nosotras, entonces visitamos el hogar del niño y con la autorización de la mamá desarrollamos 2 de las 4 actividades propuestas para ese momento y de esta manera logramos completar la finalidad que teníamos.

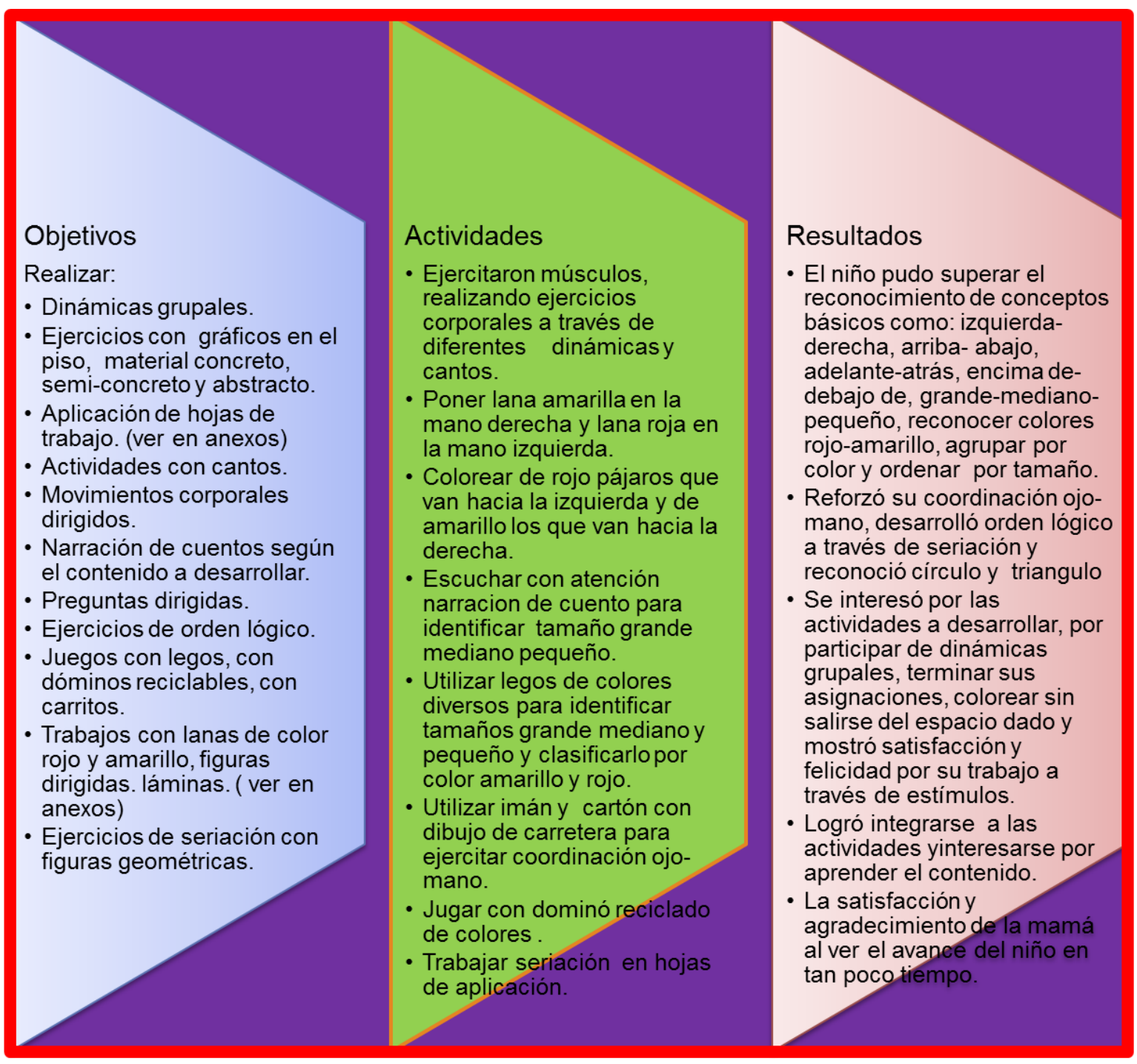

Pudimos observar y constatar que el niño respondía con deseo, dedicación y entusiasmo a todas y cada una de las actividades orientadas en los planes de acción, siendo así, nosotras nos sentimos sumamente contentas, dichosas y satisfechas de poder ser testigos de la manera en la cual el niño daba repuesta a las actividades que estaban recomendadas para él. 


\section{ANTES Y DESPUÉS}
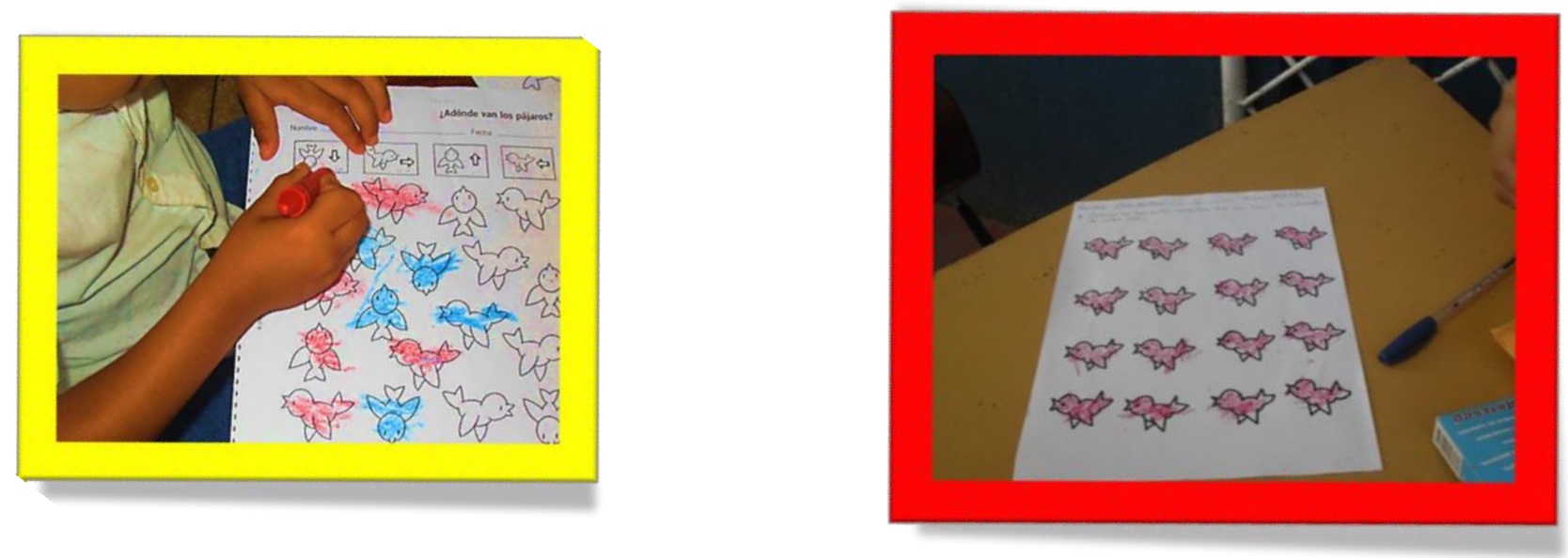

\section{CONCLUSIONES}

De acuerdo a nuestra investigación podemos concluir lo siguiente:

Esta experiencia ha sido una de las más importantes en nuestras vidas, poder apoyar a un niño y ayudarle a que mejore de manera significativa aún con pocas sesiones es inexplicable, el poder aprender de sus vidas, de su entorno familiar así como el escolar, los motivos por los cuales estos niños muestran dificultades, el acercamiento familiar y la disposición de los padres de familia, nos proporcionó muchas herramientas, objetivos y retos que debíamos proponernos para lograr un cambio en el niño.

Esta investigación y experiencia no se nos hizo fácil, a lo mejor en algún momento pudimos hacernos grandes preguntas: ¿Y ahora cómo ayudaremos a este niño? ¿Será que podamos lograr nuestros objetivos? ¿Cómo hago para que me entienda?, entre otras preguntas que se nos iban generando en el proceso, de la misma manera hubieron días muy satisfactorios y otros frustrantes pero ahí estaba el reto en poder mejorar y plantearnos otra nueva meta a lograr ya que estos fueron notorios pues el niño pudo identificar concepto de derecha, izquierda, colores amarillo, rojo, conceptos de arriba, abajo, encima de, debajo de, grande, mediano, pequeño, entre otros. Esto nos sensibilizó más con nuestro trabajo hacia los niños, nos dio el sentido de la comprensión y el compromiso a desempeñar un trabajo de calidad con estos individuos en proceso de desarrollo, siempre y cuando dándoles un seguimiento adecuado de la 
mano con el estímulo, la comprensión, el sentido de pertenencia, la dedicación, la preparación y la autoevaluación.

De acuerdo con la teoría podemos decir que el proceso lógico-matemático debe de retomarse en todos los ámbitos de aprendizaje y en todas las actividades a desarrollar pues este debe de ir en sintonía con lo antes mencionado para enriquecer los procesos cognitivos, siempre y cuando sea para reforzar o reafirmar los conocimientos de los niños de tal manera que no debemos de olvidar que para lograr una buena adquisición de los contenidos debe de retomarse el área corporal para luego pasar a lo concreto y después a lo abstracto lo cual nos llevará a un buen proceso de enseñanza-aprendizaje.

\section{REFERENCIAS}

Brissiaud, R (1993). El aprendizaje del cálculo más allá de Piaget y la teoría de conjuntos. Editorial visor, Madrid.

Conferencia Mundial sobre Atención y Educación de la Primera Infancia Moscú, Federación de Rusia 27-29 de septiembre de 2010.

Política Nacional de Primera Infancia Amor para los más Chiquitos y Chiquitas. (2011) Managua, Nicaragua

Monroy . L.(2013). El cálculo mental como estrategia para potenciar las habilidades matemáticas.

Moomaw , (2011), United States Department of Education, 2008.

Vicente, Ramírez, Dominga (2008) Estrategias metodológicas para el nivel inicial. http://www.monografias.com/trabajos61 